\title{
A critical mapping of practice-based research as evidenced by Swedish architectural theses
}

\author{
Daniela Büchler, Michael A.R. Biggs and Lars-Henrik Ståhl
}

\section{Prefatory comment}

In 2005 the Swedish government passed a Bill that broadened the scope of the Research Council (Vetenskapsrådet) to include the creative and performing arts. The Bill Research for a Better Life was the response to a three-year experimental period of ring-fenced funding in areas of national interest. In 2006 the initiatives by Vetenskapsrådet (which included funding Biggs as one of four international visiting professors in so-called practice-based research hosted in Sweden) were the subject of a quality review. The commissioning of this review showed the concern at a national level with the potential competitive advantage of establishing and exploiting a category of academic research in the creative industries (CI). Interestingly, architecture is included in these areas, unlike Britain where the research funding in practice-based research $(\mathrm{PbR})$ has mainly been in the fine and performing arts and design. The review also confirmed the existence and prevalence of $\mathrm{PbR}$ in Sweden. However, as distinct from the approach in Britain, the Swedish example suggests that this is an issue that could be investigated centrally with a view to a national definition and ultimate resolution of the problem, at least as far as the Swedish academy is concerned.

In Biggs and Büchler (2008a) the authors described an approach for determining the legitimacy of research claims about CI practices. The approach was 'criterion-based', and consisted of a systematic analysis of the foundational concepts of traditional research in order to rebuild the model in terms more fitting to the needs of areas of art, design and architectural practice. The article concluded that there were at least three necessary and sufficient conditions that could be applied to a project in order to test for the presence of $\mathrm{PbR}$. One of the aims of identifying these conditions was to provide a decision-making framework for policy makers and managers in the face of conflicting claims from the 
professional world about what constitutes research of this kind. Another aim was, if possible, to maintain a recognizable connection between the emerging model(s) of $\mathrm{PbR}$ and traditional models elsewhere in academia. The advantage of this latter strategy was to highlight what the article called the 'visibility' within the universities of both the problems and solutions arising in $\mathrm{PbR}$ in the creative and performing arts. Armed with this tool, and funded by the Swedish Institute, Büchler led a project that examined a sample of Swedish doctoral theses in architecture, in order to understand in greater detail the way in which creative practices are mobilized in architectural research. This article reports on the findings of that project.

\section{Problem Statement}

The aim of the Swedish Architectural Theses project was to enable a discussion of how creative practices contributed to the research in the sample. The objective was to identify any cases of so-called practice-based, arts-based or artistic research, generically referred to as $\mathrm{PbR}$. $\mathrm{PbR}$ is sometimes claimed to be unique to areas of creative practice in $\mathrm{CI}$, and the researchers themselves have asserted that in order for $\mathrm{PbR}$ to be a significant form of research, the artefacts produced should have an essential role in the conduct of the research, and as a result that the research could not be conducted or communicated without them (Biggs 2002). The present study aimed to clarify the characteristics of this kind of research in architecture through the analysis of a sample of doctoral theses. In the process, a selection and mapping of this sample was conducted. A critical analysis of this mapping enabled the proposition of an ontology of academic research in which the role of creative practices in research, and its possible $\mathrm{PbR}$ elements, was clarified.

The research project hypothesised that academic research containing $\mathrm{PbR}$ may constitute a novel paradigm. In an earlier article the authors characterized the genealogy of these models as arising either out of 'exploratory practice within the traditional model of academic research, [or] practice as a generator of relevant questions' (Biggs \& Büchler 2008a: 87). That initial polarization prompted this further study in order to refine the critical analysis of the situation and to identify the generic epistemological, ontological 
and methodological positions of research that has an element of creative practice, according to the schema used by Guba and Lincoln (1994: 109).

The first question the project asked was whether academic research in areas of creative practice is in some way different from traditional models of academic research that are used in other academic disciplines. The structure of the empirical part of the investigation used the sample to identify cases where traditional research criteria satisfied, or did not satisfy, the needs that the researchers themselves identified as relevant to them. When cases were identified in which the traditional research criteria did seem to satisfy the needs, these cases were further analysed in terms of whether this agreement was owing to the existence of shared concepts between the traditional model of research and CI. When cases were identified in which the traditional research criteria did not seem to satisfy the needs, these cases were further analysed in terms of whether the inadequacies of the traditional models of academic research were owing to the demands of CI-Research in academic research (Büchler, Biggs et al. 2009).

\section{Method}

Initially two searches were conducted. The first search of Swedish theses in architecture was conducted in November 2007 using the keywords:

architecture, Arkitektur Teori Filosofi, art, department of architecture, department of architecture and town planning, arts based research;

The databases and libraries searched were: Regina and Libris (Swedish National Library), KTH (Royal Institute of Technology), Stockholm University, LUP (Lund University Press), MUEP (Malmö University Electronic Publishing), Chans (Chalmers library catalogue). This search identified 212 theses. A further selection of these removed the ones that did not centrally address architecture and that did not have an online abstract in English. This resulted in 79 theses, the abstracts of which were used as the sample for the research. 
In order to enable a relevant mapping of the sample it was necessary to structure a provisional classification of models of academic research. This structuring stepped away from the particularities of the Swedish Architectural Theses project, and began to identify areas, disciplines and subjects that are adopted by research councils and universities in Sweden, the UK and Brazil. This range of countries was chosen owing to the authors having each held academic appointments in these countries. In addition to being convenient, such a heterogeneous mixture of national contexts, traditions and perspectives was also helpful in determining patterns of academic clustering of cultures of knowledge.

We have previously claimed that 'depending on how the matter is conceptualized, the distinction between $\mathrm{PbR}$ and conventional academic research can go from visible and debatable to invisible and therefore not debated' (Biggs \& Büchler 2008a: 86). How one describes something suggests how one understands it and this impacts on how things are clustered. Certain academic communities adopted specific terminology to describe what they did and how they saw what other communities did. For example, in some communities, we found that the concept of research being 'academic' was synonymous with it being 'scientific'. A critical reading of different community descriptions of academic research revealed that whilst the way in which the research activity is clustered can vary from one community to another, the fundamental understandings behind the clusters were broadly consistent.

As a result of conducting a critical analysis of the various models of academic research contained in the sample it was possible to identify three emerging meta-categories in the abstracts using procedures derived from grounded theory (Glaser \& Strauss 2007: 169). Each category contained a family of mutually comprehensible values and actions (Guba \& Lincoln 1994: 116; Biggs \& Büchler 2009). We recognized that these meta-categories, corresponding to what Goodman (1978) calls 'worldviews', could be used to organize the field of academic research. We named them: Humanities \& Human, Applied \& Social, and Natural \& Technological. 
Analysis of the sample suggested that research 'on' or 'about' architecture was sometimes produced in one or other of these three meta-categories. We also found studies 'in' architecture that were produced using theories, methods and/or frameworks from more than one meta-category. We therefore concluded that there was a fourth grouping located where the meta-categories overlapped, but that it did not constitute a fourth distinct meta-category (Figure 1). We named this overlapping category CI-Research and we speculated that $\mathrm{PbR}$ research, when present, would be found in this area. However, we noted that creative practices in CI-Research seemed to fall into two basic types: ones that were intended to have an instrumental or essential role, and ones that happened incidentally, or as some kind of by-product, to what might be regarded as the main research.

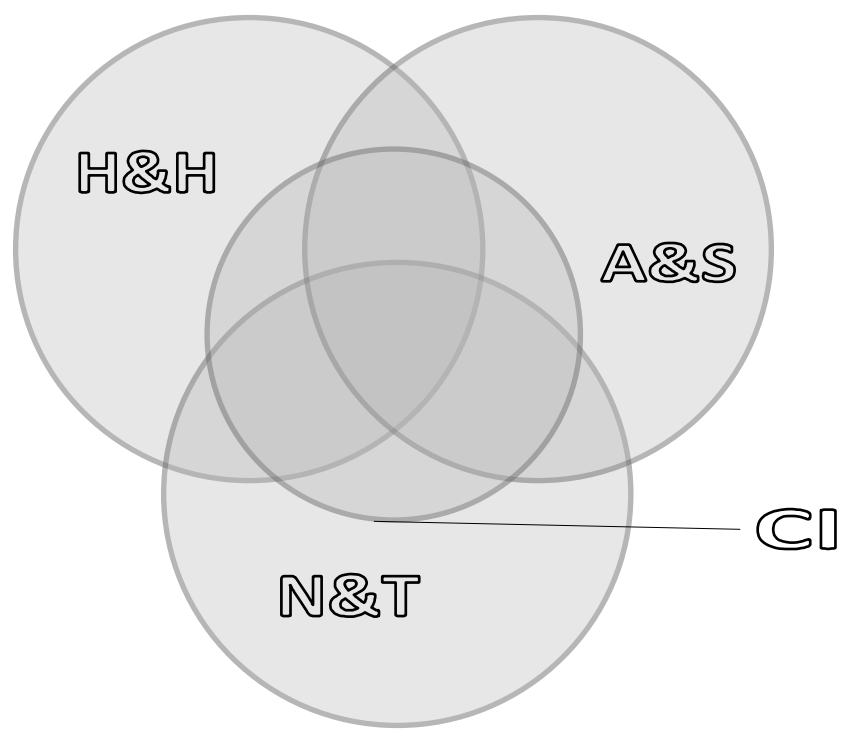

Figure 1: Two-dimensional representation of the three main cultures of knowledge in academic research (Human and Humanities; Applied and Social; Natural and Technological), expressing relationships across the research paradigms and the location of research that is conducted in areas of creative practice (CI-Research) as existing through overlapping with the three main knowledge cultures.

This analysis enabled the formulation of a graphical representation in Figure 1, of academic research that was composed of these four areas (i.e. the three meta-categories 
plus the overlapping area). Individual research projects in architecture were found to use various structures, models, frameworks and/or methods from the three meta-categories and employ creative practices, to varying degrees. Owing to basing the classification on these factors, the various cases in the sample could be mapped onto the ontology independently of the subject area claimed for the research. This was relevant because architecture is regarded as a science in some institutions, an art in others, etc., and there are correspondingly different claims and worldviews on the part of the researchers. As a result, we wanted to adopt an approach to analysing the theses that would be as independent of authorial claims and intentions as possible. We therefore preferred to group theses and activities in terms of meta-category together with the presence or absence of creative practices, rather than use subject terms such as 'architectural design'. This also allowed us to search for PbR in any subject area rather than adopting the prejudice that $\mathrm{PbR}$ was solely the province of $\mathrm{CI}$.

When mapping the sample it became clear that any interpretation would be conditioned by the epistemology in which the material could be located. This meant that in order to consider whether a thesis belonged to, say a humanities research paradigm, the epistemology of this paradigm as distinct from other paradigms had to be defined. Rather than bringing with it a problem of circularity of cause and consequence (Biggs \& Büchler 2008b: 7), this context fed an iterative reflection on and identification of some paradigmspecific characteristics.

With this need for re-conceptualization in mind, we propose that research in the Human \& Humanities category explores theoretical, historical and philosophical aspects of issues through criticism and interpretation. Studies within this culture of knowledge deal with conceptual and philosophical aspects of what, in the Applied \& Social category would be dealt with either empirically or in terms of human impact, i.e. to study what is 'perceived' and/or 'experienced' may be conducted within either of these two categories depending on whether it is considered broadly 'objectively' or 'subjectively'. Even in the event of analytical interpretation that, being conducted by an individual researcher would hold elements of subjectivity, in the Human \& Humanities category, the focus would lie on the 
object of study rather than on the reaction to, and reception of, that object. The consideration of symbols and performance of interpretation indicates Human \& Humanities, while designing the symbol system indicates CI-Research. Within our sample of 79 theses and according to our iterative analysis of the abstracts, $11 \%$ were classified as adopting the Human \& Humanities research model exclusively in their studies. Meanwhile, $57 \%$ of all studies contained some Human \& Humanities element be that method, framework, approach, etc. - to varying degrees.

We re-conceptualize Applied \& Social research as that which enhances knowledge of how society functions and how cultural values are developed and disseminated. Methods used are typically those that value the personal and subjective judgements, either of the participant or of the researcher, i.e. opinions and observations that use subjective criteria, and/or those that are participatory. The act of 'reflecting' on one's own work could be an indicator of either Applied \& Social (if there is a reflective participant) or of CI-Research (if there is a reflective practitioner/researcher). The investigation of the role of experience and creative processes would fall under this category, while the concern with process that arises from 'insider' knowledge of practice would indicate CI-Research. The use of 'interpreted' rather than 'interpretation' would connote Applied \& Social rather than CIResearch because the first presumes that the focus is on the act of interpretation, i.e. be a subject, rather than on the (perhaps less subjective) interpretation itself. The empirical consideration of emotion, experience, perception, all fall under this category while the philosophical investigation of these would be case of Human \& Humanities research. Within our sample and according to our analytical classification, 7\% of all studies adopted the Applied \& Social research model exclusively, however $62 \%$ of all studies took elements that are traditionally Applied \& Social to different degrees.

We re-conceptualize research in the Natural \& Technological category as being that which typically explores materials and/or techniques through empirical methods that imply objectivity or disinterest on the part of the researcher. The assumption is that results are analysed rather than interpreted, and therefore it is possible to produce subjectindependent results that do not rely on personal interpretation, such as occurs during 
observation or description. The development of tools, for example, can be either Natural \& Technological (when the tool itself is evaluative and can be objectified, is used to verify feasibility, weaknesses and that aims to enhance) or CI-Research (when it contributes to practice or when the development of the tool itself is a part of the research, such as when an information visualization system is created). Consideration of psychophysics and reaction to sense stimuli would denote a Natural \& Technological study of perception, while an Applied \& Social study might consider the subjective experience of stimuli. Similarly, 'interaction' could denote the user's experience and therefore be Applied \& Social, however the term is most often used in connection to hypermedia and thus suggesting Natural \& Technological. Likewise, words such as 'impact', 'quality' and 'efficiency' may indicate objective parameters and measurements and therefore Natural \& Technological. We found no studies that exclusively used the traditional Natural \& Technological research model, while $45 \%$ of all studies used elements of this kind of research model to different degrees. It is perhaps interesting that $90 \%$ of all the studies that adopted Natural \& Technological research elements also contained CI-Research elements of practice and, furthermore, $30 \%$ of that practice was considered to be of the PbR kind, as explained below.

\section{Creative Practices and Practice-based Research}

In the construction of the working $\mathrm{PbR}$ ontology, our interest was to see whether cases could be differentiated between those merely having creative practice, and those in which the creative practice had an instrumental role. We were interested in this difference because it seemed to us that, employing the Principle of Parsimony, there was no need to create a new category of research if it was already catered for in existing classifications. Therefore, we hypothesised that $\mathrm{PbR}$ occurs when there is a unique and inner synergy between practice and the traditional academic research elements, namely question, method, answer and audience (Biggs \& Büchler 2008b). In order to focus on these distinctions, we narrowed the sample further by extracting theses that contained an element of creative practice. This reduced the sample from 79 to 17 theses. These 17 were theses that either made claims about $\mathrm{PbR}$ or contained an element of practice. Upon 
re-reading these abstracts, issues arose in eight examples both about the claim that was being made for the role of practice in the research and about the role of practice itself. We therefore analysed the full theses of these eight examples and conducted semi-structured interviews in Lund and Stockholm with the researchers themselves and their supervisors.

Creative practice was found to play different roles in the theoretical argument. These roles ranged from practice serving to illustrate, demonstrate, prove or explore theoretical constructs, and were often not essential to the argument but added value to it in some way. However, there was also a role for practice that went beyond and somehow contributed to the generation of knowledge, and could therefore be argued to be essential to the argument. In these cases, without the practice element, something would be lost. This observation meant that practice could contribute in terms of process and creative insight, but that practice could also create knowledge of a different kind. Although it is outside the remit of this study, and still under discussion worldwide what this 'kind' would be that distinguishes incidental practice from consequential practice (Eisner 1990; Phillips 1995; Candlin 2000; Finley 2003; Barfield \& Quinn 2004; Sullivan 2005; Macleod \& Holdridge 2006; Mäkelä \& Routarinne 2006; Biggs \& Büchler 2008b), the observation reinforced our decision to create two different labels for research with an element of practice: $\mathrm{CI}-\mathrm{Research}$ and $\mathrm{PbR}$. As a result of making the representation in Figure 1, and observing that practice and making occurs in many disciplines, we concluded that the mere presence of practice is not in itself the defining characteristic of $\mathrm{PbR}$.

While there is an assumption that practical and non-textual activities are related under a 'non-traditional activities' banner, this is not a rule and there are of course many examples of the use of creative practices in research that is conducted in the three metaareas. However, in CI, there is often a claim that any creative practice that is conducted by the creative practitioner in the process of, or towards, academic research, is itself either the contribution to knowledge or instrumental to that knowledge. The distinctions between the essential and discretionary role of practice have informed the definition of the next two categories of research, i.e. CI-Research and PbR. 
Practice can contribute to research in CI in different ways, and this is why in Figure 2 we introduced a third dimension. This enables us to represent our claim that discretionary practice may occur in any of the three meta-categories, as well as in the intersections that form CI-Research. There is a distinction between this everyday practice that is represented on the two-dimensional plane, and the instrumental $\mathrm{PbR}$ practice that is represented in the third-dimension. In the former, practice is not essential to the argument while in the latter, practice is either essential to the argument or contributes something that could not have been contributed otherwise.

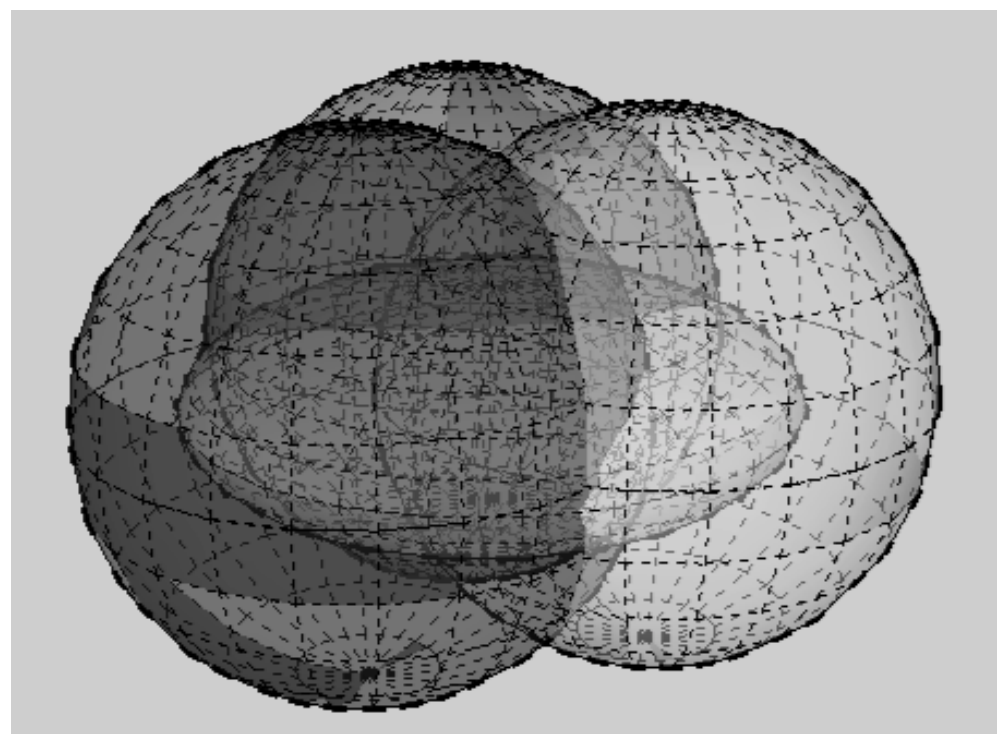

Figure 2: Three-dimensional representation of research activity across the three main cultures of knowledge and research in CI that presents an element of practice, including the third $(\mathrm{PbR})$ dimension where creative practice generates knowledge that is unique and essential to the contribution to knowledge.

CI-Research tends to be cross-, inter- and/or trans-disciplinary and use, by definition, a range of methods, theory and practices from other areas (Biggs \& Büchler 2011b). Applied research could be either CI or Natural \& Technological depending on whether the act of applying and testing out the research contributed to that research, or whether merely the resulting data was used, i.e. a focus on the findings would denote the latter while focus on the personal and/or creative process would denote former. Because our sample came from studies on, about and in architecture, it would be expected that the 
bulk of these could be grouped under the CI-Research category. However, as we have chosen to define CI-Research in terms of the presence and role of practice in the research, we have found that actually only $71 \%$ of all 79 studies responded to the requirements of the CI-Research category, while the rest were either exclusively Human \& Humanities (11\%) or exclusively Applied \& Social (7\%), or combinations of two or three of the meta-categories (11\%). While expressive, this $71 \%$ of CI-Research was further differentiated, and it was found that only $15 \%$ (i.e. the 8 examples where the full theses were studied and the interviews were conducted) was potentially of the PbR kind.

Research is of the PbR kind when either: (1) creative and/or non-traditional practice is an integral part of the development of the research or (2) the conceptualization of the problem and solution to that problem is born out of the practice or (3) there is no conscious distinction or separation between research and practice on the part of the researcher. In (Biggs \& Büchler 2011a) we develop the notion of there being conscious and unconscious actions as a consequence of one's worldview that determines what is thinkable, visible and what one is aware of. The 'unconsciousness' on the part of the researcher that research and practice are separate can be contrasted to the notion of 'applied' research, which suggests a planned contribution. When the researcher sets out to produce a $\mathrm{PbR}$ doctorate, there is an awareness that this is as opposed to a non- $\mathrm{PbR}$ route. Although it was found that all $\mathrm{PbR}$ research was also CI-Research (i.e. presented an element of practice), not all CI-Research was also $\mathrm{PbR}$. Indeed, it was found that in the cases where there was an intention to include practice in the research, the study usually turned out to be CI-Research rather than $\mathrm{PbR}$. In the 79 theses, only five were classified as $\mathrm{PbR}$, representing $6 \%$ of the total. It is worth highlighting that this expressively small percentile is dependent on our particular definition of the category of $\mathrm{PbR}$.

\section{Three strategies and two positions}

As a result of differentiating CI-Research as a sub-category in which the practitionerresearcher attempts to involve their practice in the research, we were able to ask a second 
research question: how do researchers attempt to combine creative practice as an element of academic research? By observing the sample in terms of the role of creative practice, we identified three strategies that were adopted for conducting research in architecture, and hence potentially in CI more generally.

The 'unconsciousness' requirement implies that the creative practice and academic research communities can be regarded as a single community or as two distinct communities, and this distinction can be seen as being possible or impossible to define, desirable or undesirable to bridge. In Figure 3, Position 1 represents the view that, although there are two distinct communities, the practitioner only inhabits the creative practice community and produces both practice and research in terms of that set of community values. In this case transposition of one community to another is undesirable and unnecessary for the production of academic research with practice. Position 2 is an example of the unconsciousness of the existence of two distinct communities, where there is a single set of values that address both practice and research and an undifferentiated attitude towards production.

When the communities are seen as distinct and bridging between these is seen as possible and desirable for some reason, it is necessary for the researcher to adopt a strategy in order to include creative practice in academic research. These strategies are adopted/created as a response to the assumption that there are two distinct communities the $\mathrm{CI}$ and the academic - and that therefore when conducting research in CI, compensatory strategies are necessary to ensure that the values and requirements of both communities are represented. In Figure 3, the circles represent that the values and consequent actions and activities that are developed by that community are seen as something that should be upheld and preserved. When the values and practices of a community are taken to another community, represented by the arrow, it is because the researcher wishes to transform the other community by changing in some way the values or actions of that community. In Strategies 1, 2 and 3 there is an attempt to bridge one community and the other. 


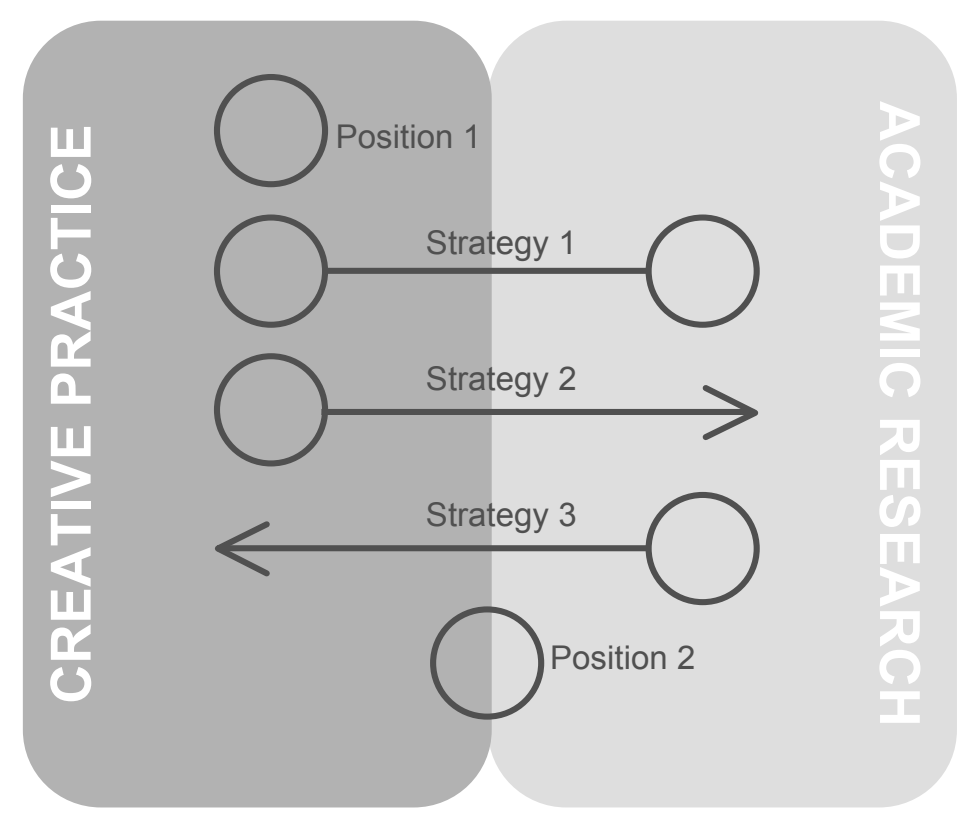

Figure 3: Diagrammatic representation of the two communities - creative practice and academic research - the two positions and three strategies.

The strategies can be described in terms of whether the researcher will uphold (represented in the diagram by a circle "O") or transform (represented in the diagram by an arrow head " $\leftarrow$ " or “ $\rightarrow$ ") element(s) from traditional academic models in order to produce research in areas in which there is no single accepted/specific research model. That which a researcher sees as worthy of being preserved is taken holistically because it is seen as indisputably relevant and/or essential either for the production of academic research in any area, or for the production of creative practice as defined by the community of practice. To uphold and preserve therefore implies that the researcher values what is being preserved and should remain untransformed. The element that is taken should not be transformed, however when it is used or applied in the new community it should transform that community either by supplying the essential academic research model or by validating the non-traditional element that is of value in that community. Therefore, the strategies are approaches to mediate the interests of the two communities.

Strategy 1 describes that researcher who transits from one community to another, and depending on what community the researcher is in, so he/she takes and uses that 
community paradigm completely, i.e. values and research model, without transforming either community. When adopting Strategy 2, the researcher takes his/her own practices and/or values and uses it in a traditional academic context in an attempt to transform accepted academic practices so that their practice is recognized as academically valid without having to be transformed. Strategy 3 describes the researcher who takes traditional academic models and uses these in order to transform his/her practice and, by associating that practice to what is seen as being of value in the academic community, the researcher attempts to validate elements of his/her, now, transformed practice.

According to the rationale of Figure 3, $\mathrm{PbR}$ would not be a strategy but would be the research that is produced in Position 2. This is because a strategy is by definition a plan for bridging two communities, whereas a researcher in Position 2 does not recognize the two communities as distinct but as a single and integrated one, and as a result no strategy is needed. The descriptors 'uphold' and 'transform' structure the construction of a research strategy, i.e. an interim research model because a definitive one is as yet unavailable. On the other hand, the concept of $\mathrm{PbR}$ is research that is a consequence of a particular (and unique) worldview. In this sense, $\mathrm{PbR}$ is not constructed but emerges as a result of a coherent relationship between worldview and research model much in the way that traditional research models have been structured.

\section{Conclusions}

The investigation sought to make a contribution to the debate around so-called practicebased by trying to identify examples in a sample of Swedish doctoral theses in architecture. However, since the nature of $\mathrm{PbR}$ is contested, several procedures had to be undertaken to clarify the criteria for identification. Rather than relying on the claims of the individual doctoral candidates themselves, we adopted a model which described broad subject areas in terms of cultures of knowledge. Within a culture of knowledge, there is agreement on the ontological, epistemological, and methodological conditions (worldview), and hence agreement on what would constitute effective actions in pursuit of new knowledge. Within this model, three meta-level categories were identified, none 
of which was specific to research in the creative industries, and each of which had the potential to contain creative practice. The sample was mapped and the theses containing creative practice were found to be distributed across all categories. At the intersection of these three meta-categories, a fourth was identified as containing theses with creative practice. It was noted that the function of the creative practice in the research in this category was varied. We refined the model to differentiate between types of creative practice that were essential in the research, and types that were discretionary. This led to the first conclusion of the investigation: that the mere presence of creative practice is not necessarily an indicator of the presence of $\mathrm{PbR}$.

In order to differentiate the various roles of creative practice in research, the theses containing such practice were further analysed in terms of the strategies adopted for managing the contribution that the practice made to the research. Three strategies and two positions were identified, which were described in terms of the researchers' view on creative practice and academic research as communities, and the value system that they adopted when producing academic research with elements of practice. This led to the second conclusion of the investigation: that the use of creative practice can mask a lack of integration between the aims of the research and the actions that are undertaken. Only when there is coherence between these aims and actions can the practice usefully contribute to the research, and only when this coherence also maps onto the community values can this research be of the PbR kind. Only 6\% of the sample was found to be potentially $\mathrm{PbR}$, whilst the rest either adopted these masking strategies, or did not contain creative practice.

This research contributes to the debate by clarifying the ontology of $\mathrm{PbR}$. To avoid redundancy, $\mathrm{PbR}$ must not duplicate types of research that are already established elsewhere. To ensure meaningfulness, $\mathrm{PbR}$ must ensure that its actions are in accord with the interests, aims and worldview of the community. To establish the possibility of PbR as a new paradigm, examples must be found of research that meets these requirements. In this investigation, five cases were identified that seemed to do so. 


\section{References}

Biggs, M.A.R. (2002). The Role of the Artefact in Art and Design Research. International Journal of Design Sciences and Technology 10 (2): 19-24.

Biggs, M.A.R. \& D. Büchler (2008a). Architectural Practice and Academic Research. Nordic Journal of Architectural Research 20 (1): 83-94.

--- (2008b). Eight Criteria for Practice-based Research in the Creative and Cultural Industries. Art, Design and Communication in Higher Education 7 (1): 5-18. 10.1386/adch.7.1.5_1

--- (2009) Supervision in an Alternative Paradigm. TEXT: Journal of Writing and Writing Courses Special Issue 6, 1-14 http://www.textjournal.com.au/speciss/index.htm [accessed on 17 February 2010].

--- (2011a). Communities, Values, Conventions and Actions. In: M. Biggs \& H. Karlsson, Eds. The Routledge Companion to Research in the Arts. London, Routledge: 82-98.

--- (2011b). Transdisciplinarity and New Paradigm Research. In: I. Doucet \& N. Janssens, Eds. Transdisciplinary Knowledge Production in Architecture and Urbanism. Dordrecht, Springer Verlag.

Büchler, D., M.A.R. Biggs \& L.-H. Ståhl (2009). Areas of Design Practice as an Alternative Research Paradigm. Design Principles and Practices: an international journal 3 (2): 327-338.

Glaser, B.G. \& A.L. Strauss (2007). The Discovery of Grounded Theory: strategies for qualitative research. London, Aldine Transaction.

Goodman, N. (1978). Ways of Worldmaking. Indianapolis, Hackett Publishing.

Guba, E. \& Y. Lincoln (1994). Competing Paradigms in Qualitative Research. In: N. Denzin \& Y. Lincoln, Eds. Handbook of Qualitative Research. London, Sage Publications: 105-117. 